\title{
Special issue on software quality of advanced software applications
}

\author{
Lefteris Angelis $^{1} \cdot$ Tomas Bures $^{2}$
}

Published online: 27 April 2020

C) Springer Science+Business Media, LLC, part of Springer Nature 2020

This issue of the Software Quality Journal is devoted to studies on the quality of software in various advanced applications in business, industry, and everyday life. Following an open call and a thorough review process, the articles accepted for publication cover various aspects relevant to the generic topic. Various areas are represented in the published papers, such as embedded systems, gap analysis, component-based software engineering, open source software analysis, software management, bug reports, and domain-specific modeling. Moreover, different qualitative and quantitative methodologies are used such as empirical studies, questionnaires, prioritization techniques, statistical analysis, software repositories mining, recommendation systems, text analysis, and systematic literature mapping.

This issue consists of 7 papers that are briefly discussed as follows:

The article by D. Sas and P. Avgeriou entitled "Quality attribute trade-offs in the embedded systems industry - An exploratory case study" deals with challenges in the embedded systems industry and specifically with the study of practices, processes, and tools concerning the management of run-time and design-time quality attributes as well as the trade-offs among them from the perspective of embedded systems software engineers. The authors use qualitative data from interviews and a focus group session with participants from six different companies and their analysis reveals interesting preferences, practices, difficulties, and risks related to software qualities and their trade-offs, along with needs for automated tools.

In "A Model Driven Engineering approach for supporting Questionnaire-Based Gap Analysis Processes through Application Lifecycle Management Systems" by D. Amalfitano, V. De Simone, S. Scala, and A. R. Fasolino, the authors deal with questionnaire-based gap analysis, a process for evaluating the gaps between implemented software and quality requirements through questionnaires. The paper proposes the utilization of application lifecycle management

Tomas Bures

bures@d3s.mff.cuni.cz

Lefteris Angelis

lef@csd.auth.gr

1 School of Informatics, Aristotle University of Thessaloniki, 54124 Thessaloniki, Greece

2 Charles University in Prague, Malostranske namesti 25, 11800 Prague, Czech Republic 
and model driven engineering to support the aforementioned processes. The quality of the suggested approach is applied and evaluated by a case study in the automotive industry.

In "Component Attributes and their Importance in Decisions and Component Selection" by P. Chatzipetrou, E. Papatheocharous, K. Wnuk, M. Borg, E. Alégroth, and T. Gorschek, the authors deal with component-based software engineering and investigate the characteristics that are more important in the selection of new components. They used data from a prioritization procedure with participants from industry and the statistical analysis, involving multivariate methods and hypothesis tests, revealed the most important attributes, the relations between them and the differences in the prioritization between large and small organizations.

The article "An Automated Approach to Assess the Similarity of GitHub Repositories" by P. T. Nguyen, J. D. Rocco, R. Rubei, and D. D. Ruscio deals with mining open source repositories for finding similar software projects. The ultimate goal is the development of highquality software through learning from mature and successful projects. The novel methodology proposed by the authors, computes similarities among software projects and can be used to reduce the development effort and improve various phases of the software life cycle such as implementation and maintenance.

The article "Structured Information in Bug Report Descriptions - Influence on IR-based Bug Localization and Developers" by M. Rath and P. Mäder relies on textual similarity of the bug report description and the source code files. The paper systematically analyzes the influence of structured information on information retrieval (IR)-based techniques. The empirical study is based on 7334 bug reports.

The article by C. Tjortjis entitled "Mining Association Rules from Code (MARC) to Support Legacy Software Management" presents a methodology that performs code analysis, association rule mining and rule grouping. The goal is to capture program structure so as to facilitate system understanding and support the software management. The methodology was evaluated on COBOL programs showing its usefulness regarding program comprehension by using source code only, where domain knowledge and documentation are either unavailable or unreliable.

The article entitled "A Systematic Mapping Study on Best Practices for Domain-Specific Modeling” by G. Czech, M. Moser, and J. Pichler uses a literature search in order to identify studies reporting best practices on domain-specific modeling. The systematic analysis of the retrieved studies results in a comprehensive compilation of best practices, aiming to act as a starting point, able to facilitate and guide the development, and application of domain-specific modeling solutions.

The special issue was preceded by the 44th Euromicro Conference on Software Engineering and Advanced Applications (SEAA 2018), held at Prague, Czech Republic, August 29-31, 2018. The articles have undergone rigorous peer-review according to the journal's high standards. From the 20 originally submitted articles in this issue, only 7 were finally accepted for publication, i.e., the acceptance rate was $35 \%$.

Collectively, the papers comprising the current issue are indicative of the wide and diverse range of topics being investigated in the generic field of software quality in advanced applications and provide a detailed compilation of different methods involved in the related research.

Publisher's note Springer Nature remains neutral with regard to jurisdictional claims in published maps and institutional affiliations. 